\title{
Escala de Avaliação da Autonomia Profissional dos Professores: adaptação e propriedades psicométricas
}

\author{
Sílvia Fernandes do Vale ${ }^{1}$, Regina Heloisa Maciel(D) \\ Universidade de Fortaleza, Fortaleza-CE, Brasil \\ Mateus Estevam Medeiros-Costa (D) \\ Universidade Federal do Rio Grande do Norte, Natal-RN, Brasil
}

\section{RESUMO}

O presente artigo objetiva adaptar e apresentar as propriedades psicométricas da Escala de Avaliação Autonomia Profissional dos Professores (EAP) para o contexto brasileiro. A escala foi aplicada coletivamente nas escolas em 407 professores da rede pública de educação do município de Mossoró-RN. Em sua maioria mulheres (73,8\%), com idade média de 44,33 \pm 8,37 anos. Análises fatoriais exploratórias realizadas no software Factor corroboram a existência de dois modelos, mas a estrutura composta por seis fatores demonstrou melhor ajuste $\left(\chi^{2}=27152.374, \mathrm{CFI}=1,001 ; \mathrm{TLI}=1,001 ; \mathrm{RMSEA}=0,000\right)$, em comparação à solução tridimensional. A consistência interna conferida pelo alfa de Cronbach mostrou-se satisfatória. São sugeridos estudos que confirmem uma estrutura fatorial generalizável para fins de replicação.

Palavras-chave: professor; autonomia; educação; psicologia do trabalho; análise fatorial.

\section{ABSTRACT - Teacher Professional Autonomy Scale: adaptation and psychometric properties}

This study aimed to adapt the Teacher version of the Professional Autonomy Scale (PAS) for the Brazilian context and investigate its psychometric properties. The scale was applied collectively with 407teachers of schools of the public education system of the municipality of Mossoró-RN. The participants were mostly female (73.8\%), with a mean age of $44.33 \pm 8.37$ years. Exploratory factor analyses performed using the Factor software corroborated the existence of two models, with the six-factor structure showing the best fit $\left(\chi^{2}=27152.374\right.$, CFI=1.001; TLI=1.001; RMSEA=0.000), compared to the three-dimensional solution. The internal consistency conferred by Cronbach's alpha was satisfactory. Studies are suggested to confirm a generalizable factor structure for replication purposes.

Keywords: Teacher; Autonomy; Education; Work psychology; Factor analysis.

RESUMEN - Escala de Evaluación de la Autonomía Profesional del Profesorado: adaptación y propiedades psicométricas

Este artículo tiene como objetivo adaptar y presentar las propiedades psicométricas de la Escala de Evaluación de la Autonomía Profesional del Profesorado (EAP) para el contexto brasileño. La escala se aplicó colectivamente en las escuelas con 407 docentes del sistema de enseñanza pública del municipio de Mossoró-RN. Participaron en su mayoría mujeres (73.8\%), con una edad media de $44.33 \pm 8.37$ años. Los análisis factoriales exploratorios realizados en el Software Factor corroboran la existencia de dos modelos, pero la estructura compuesta por seis factores mostró el mejor ajuste $\left(\chi^{2}=27152.374, \mathrm{CFI}=1,001 ; \mathrm{TLI}=1,001 ; \mathrm{RMSEA}=0,000\right)$, en comparación con la solución tridimensional. La consistencia interna conferida por el alfa de Cronbach resultó ser satisfactoria. Se sugieren estudios para confirmar una estructura de factores generalizable para fines de replicación.

Palabras clave: Docentes; Autonomía; Educación; Psicología del trabajo; Análisis factorial.

Desde a década de 1980 o sistema educacional brasileiro vem adotando políticas neoliberais, engajadas na "mercantilização da educação". Atualmente já se tramitam propostas sobre a terceirização e privatização da escola pública. Discursos sobre "eficácia" e a "eficiência" ganham espaço nas instituições de ensino e modelos de gestão respaldados no controle como sistemas de avaliação externa, implantação de projetos determinados externamente entram em cena, propiciando uma autonomia docente controlada (Fernandes \& Barbosa, 2014).

O modelo de gestão pautado no controle prescreveu o taylorismo-fordismo e se expandiu na década de 1990 com a reestruturação produtiva por meio do modelo flexível de trabalho, sendo introduzido nos diversos contextos laborais, inclusive na tarefa docente (Souza, et al., 2015).

${ }^{1}$ Endereço para correspondência: Rua São Gabriel, 250, apto 201, Bairro Cocó, 60135-450, Fortaleza, CE. E-mail: sfvale@hotmail.com; sfvaleunifor@gmail.com.br Artigo derivado da Dissertação de mestrado da Sílvia Fernandes do Vale com orientação da Regina Heloisa Mattei de Oliveira Maciel, defendida em 2013 no programa de pós-graduação Stricto Sensu em Psicologia da Universidade de Fortaleza (UNIFOR). 
Nesse cenário, os sujeitos são coagidos a trabalhar dentro de um quadro, em que, de um lado se incentiva a exercer a autonomia, do outro são instituídas diretrizes de controle que regem a tarefa do professor, as quais inibem a autonomia do professor e a capacidade de concepção, podendo atingir sua integridade física e emocional $\mathrm{e}$, consequentemente, ocasionar o adoecimento (Martins et al., 2014). Nessa perspectiva, Filho (2020) afirma que a perda da autonomia e autoria nas atividades desenvolvidas pelos professores, as metas de produtividade, entre outras são causas de sofrimento psicológico/adoecimento derivados do trabalho docente.

Lessard (2006, p. 145) "esclarece que vivenciamos a era da autonomia prescrita e da iniciativa obrigada ou forçada". A relação entre controle e autonomia se dá por meio da associação de forças específicas junto a docentes e políticas educacionais. Já que o sistema de ensino estabelece reformas educacionais que emergem modos de controle sob o trabalho do professor, e se há "por parte do Estado a imposição de controle externo por meio dessas regulamentações, igualmente existe por parte dos docentes uma resistência a essa perda de autonomia" (Souza et al., 2015, p. 137). Destaca-se que o controle do trabalho docente é compreendido como o arcabouço de políticas públicas e de práticas administrativas que visam nortear o trabalho do professor.

Por outro lado, a palavra "autonomia" no trabalho vem sendo explorada por diversas abordagens teóricas, podendo ser compreendida como um novo meio de organizar o trabalho, além de contribuir para a manifestação da inteligência prática, possibilitando que o trabalhador crie formas mais eficazes de desempenhar suas atividades (Moraes et al., 2012). A priori, a autonomia já era vista como uma ação capaz de modificar a prescrição da tarefa, de modo a moldá-la ao real do trabalho (Ferreira, 2008).

A autonomia do professor se apresenta como um conceito abrangente que perpassa diferentes aspectos (filosóficos, psicológicos, sociológicos e históricos-políticos), campos e tipos de restrições, visto que, ao analisar esse fenômeno, essas múltiplas dimensões devem ser levadas em conta (Erss et al., 2016; Wilches, 2009). Por esse motivo, o conceito de autonomia traçado no presente estudo envereda por uma perspectiva histórico-dialógica, como emancipação, superação das distorções ideológicas e consciência crítica (Contreras, 2002). Inteirando, Souza et al. (2015) toma a autonomia profissional do professor como a capacidade de pôr em prática diferentes etapas do saber fazer pedagógico e a capacidade de tomada de decisões metodológicas.

A prática autônoma não é tão simples, já que a autonomia diz respeito à habilidade que o sujeito possui de agir por si mesmo, de tomar decisões conscientes e com criticidade, além de assumir com seriedade os seus atos. Geralmente as práticas pedagógicas são embasadas por determinação de regras em vez de momentos que permitam condições de diálogo, em que os profissionais da educação em suas relações uns com os outros e com o professor possam se assumir como seres sociais e históricos, pensadores, comunicantes, transformadores. As temáticas em sala de aula não são tratadas como se deve, são discursadas. A tarefa do educador é alicerçada no poder sob o aluno, vestígios da "inexperiência democrática" vivenciada no decorrer dos anos pela maioria da população (Freire, 1996).

Foi entre os anos 1960 que surgiram os primeiros indícios sobre a importância da autonomia docente. Consequentemente na década seguinte foram validados os primeiros instrumentos, mas a escala de maior visibilidade naquela ocasião era a Sense of Autonomy Scale (SAS) (Charters Jr., 1976; Friedman, 1999). Posteriormente, Chauvin e Ellett (1993) utilizaram a Attitudes of Professional Autonomy (APA) numa amostra composta por 1.921 professores e, após o processo de validação, a escala apresentou uma estrutura bidimensional. Estrutura semelhante já encontrada em outras categorias, como administradores, advogados, bibliotecários, profissionais da enfermagem, engenheiros e médicos (Forsyth \& Danisiewicz, 1985).

Tanto a SAS (Charters Jr., 1976), quanto a APA (Forsyth \& Danisiewicz, 1985; Chauvin \& Ellett, 1993) são escalas estruturadas numa perspectiva individualista, as quais exploram apenas dimensões acerca da desconfiança, influência no trabalho, controle, demandas organizacionais e limitações pedagógicas. Deixando de fora questões referentes ao envolvimento do educador nas principais práticas escolares e nas políticas públicas (Friedman, 1999). Afora, outros instrumentos vêm sendo validados, os quais traçam novas perspectivas e dimensões sobre a autonomia docente (Friedman, 1999; Pearson \& Moomaw, 2005 Khezerlou, 2013; Ulas \& Aksu, 2015; Veiga et al., 2003; Yu-hong \& Ting, 2012).

Vale ressaltar as mudanças que envolvem o sistema educacional e os diferentes domínios que perpassam o trabalho do professor na atualidade, como ensino e avaliação, desenvolvimento curricular, desenvolvimento profissional e funcionamento escolar, nesse sentido, torna-se fundamental a construção de instrumentos que envolvam as novas demandas educacionais. Evers et al. (2017) construiu uma escala numa perspectiva mais ampla, a partir de uma revisão de literatura, que pode ser utilizado em diferentes contextos educacionais para medir o comportamento autônomo do professor, a qual abrange quatro dimensões: o trabalho na sala de aula, implementação de currículo, participação na tomada de decisões na escola e o desenvolvimento profissional. Para atender o contexto educacional online que ganha força neste século XXI, Nayernia (2020) desenvolveu uma escala com sete categorias que contribuem para o apoio da autonomia do professor.

Há aqueles estudos (Derakhshan et al., 2020; Evers et al., 2017; Vangrieken et al., 2017; Worth \& Brande, 2020) que analisam a relação entre a autonomia e outros 
construtos, porque a autonomia do professor é uma variável de grande importância no contexto educacional, pois ela contribui para a legitimação do status do professor no âmbito coletivo e social, ela é crucial para a tomada de decisões e para o sucesso, para o processo de colaboração e o desenvolvimento profissional docente. Afirmam os autores que a autonomia está fortemente correlacionada com vários fatores, como a satisfação no trabalho, o processo de colaboração, a administração da sua carga de trabalho, a quantidade de tempo de lazer, a intenção de permanecer na carreira docente. A autonomia percebida pelo professor desempenha um papel positivo no que diz respeito ao desenvolvimento profissional, bem como apresenta uma associação negativa com estresse, esgotamento e Burnout (Pearson \& Moomaw, 2005; Skaalvik \& Skaalvik, 2014).

Os estudos citados justificam a importância de adaptar e validar uma escala de autonomia do professor no contexto brasileiro, pois a esta se apresenta como uma variável-chave na motivação dos professores em desenvolver suas atividades, no desempenho discente, bem como permanecer ou deixar a docência. Como também, no Brasil, as comunicações científicas sobre a autonomia profissional do professor são incipientes e, na maioria dos casos, são estudos teóricos reflexivos ou pesquisas de abordagem qualitativa. Os estudos sobre a autonomia junto a outros trabalhadores são mais frequentes (Ferreira, 2008; Moraes et al., 2012), sendo que, entre os profissionais de enfermagem, essa discussão é mais consolidada (Przenyczka et al., 2012; Santos et al., 2012).

Pela ausência de instrumentos de medidas válidos e confiáveis, este estudo tem por objetivo adaptar a Escala de Avaliação da Autonomia Profissional dos Professores (EAP) para o contexto brasileiro, apresentando suas propriedades psicométricas e consistência interna. A hipótese deste estudo é de que o modelo da EAP de seis fatores apresentará ajuste satisfatório para explicar a autonomia docente no contexto brasileiro em virtude do trabalho multifacetado do professor.

\section{Método}

\section{Processo de Adaptação da EAP}

Inicialmente, dois experts independentes da área da avaliação psicológica foram os responsáveis pela verificação das questões semânticas, idiomática, conceituais, contextuais e linguísticas da versão da língua original para o idioma-alvo e, desse processo, duas versões da EAP foram apresentadas. A síntese das duas foi realizada e a avaliada por um grupo de especialistas $(N=10)$, os quais verificaram a semelhança entre dois itens da escala, sugerindo, desse modo, a retirada de um deles. Após excluir o item " 20 - Tenho poder para estabelecer os critérios de avaliação do rendimento escolar dos alunos", a versão sintetizada - contendo 30 itens - foi avaliada por um grupo-alvo $(N=13)$ e por quatro experts na avaliação psicométrica que deram parecer favorável e assim a escala foi considerada pronta para uso (Borsa et al., 2012; Borsa $\&$ Seize, 2017).

\section{Participantes}

A amostra não probabilística foi composta por 407 professores da rede pública de educação do município de Mossoró. Em sua maioria são mulheres $(73,8 \%)$, com idade média de 44,33 $\pm 8,37$ anos, variando de 20 a 68 anos, casadas ou que vivem em união consensual estável (56,9\%), com filhos (74\%). A carga horária semanal dos professores varia entre $30 \mathrm{~h}$ e $45 \mathrm{~h}(52,7 \%)$, tendo uma média de tempo de trabalho de 19,91 \pm 9,03 anos. A amostra ficou distribuída: professores da educação infantil $(N=62 ; 15,4 \%)$; professores do ensino fundamental dos anos iniciais $(N=143 ; 35,4 \%)$ e finais $(N=72 ; 17,8 \%)$; como também professores da educação de jovens e adultos $(N=42 ; 10,4 \%)$ e do ensino médio $(N=85 ; 21 \%)$.

\section{Instrumentos}

Os instrumentos utilizados foram:

Questionário sociodemográfico. Foram recolhidas informações referentes ao sexo, idade, estado civil, número de filhos, formação educacional, nível de ensino, número de horas semanais e tempo de trabalho.

Escala de Avaliação da Autonomia Profissional dos Professores - EAP (Veiga et al., 2003). O aporte teórico e os itens selecionados para essa medida tomaram como referência o estudo que apresenta o processo de validação da escala Appropriate Teacher Work-Autonomy (ATA) de Friedman (1999). Em decorrência, a EAP é distribuída em 31 itens, com respostas tipo Likert, variando de 1 (Nenhuma autonomia) a 6 (Total autonomia), com uma estrutura multidimensional subdivida em seis fatores distintos: Autonomia na Formação de Turmas, suas Condições e Normas Escolares; Autonomia nas Temáticas de Ensino; Autonomia na Formação Profissional; Autonomia no Relacionamento com os Encarregados de Educação; Autonomia no Ensino e na Avaliação do Rendimento Escolar; e Autonomia na mudança e no Desenvolvimento Curricular. A EAP no seu processo de validação junto a professores portugueses apresentou um alfa de Cronbach $=0,95$, propriedades psicométricas adequadas e índices de confiabilidade.

\section{Procedimentos}

\section{Coleta de Dados}

Inicialmente foi desenvolvida uma pesquisa piloto em duas escolas públicas de Mossoró-RN junto a 33 professores. Em seguida, foram selecionadas escolas de diferentes bairros da cidade, totalizando 61 escolas (estaduais e municipais) e 10 unidades de educação infantil. Ao chegar à escola, era apresentado ao diretor e/ou coordenador pedagógico o termo de aprovação do projeto pelo Comitê de Ética. Assim, o contato com os professores 
se deu por meio de visitas às escolas. Os instrumentos foram aplicados de forma coletiva na sala dos professores ou de reuniões, geralmente no horário de intervalo ou durante os encontros de formação e/ou reuniões pedagógicas. O presente estudo cumpriu com as orientações éticas e foi aprovado pelo Comitê de Ética da Universidade de Fortaleza (UNIFOR).

\section{Análise dos Dados}

O banco de dados gerado no IBM-SPSS 22.0 foi submetido ao software Factor versão 10.8.04 para realização da análise fatorial exploratória (AFE). A adequabilidade da amostra foi avaliada pelo teste de esfericidade de Kaiser-Meyer-Olkin (KMO) e Bartlett. Para retenção dos fatores, seguiu-se três parâmetros: construtos teóricos, ou seja, a EAP é estruturada por seis dimensões (Veiga et al., 2003); autovalores superiores a 1, como sugerido por Guttman-Kaiser; e Análise Paralela (AP) (Timmerman \& Lorenço-Seva, 2011). A rotação oblíqua Promin foi escolhida para verificação das cargas entre fatores (Lorenzo-Seva, 2013; Damásio \& Dutra, 2017).

A adequação da EAP foi conferida mediante os seguintes índices de ajustes: índice de ajuste comparativo (CFI), índice de Tucker-Lewis (TLI) e erro médio quadrático de aproximação (RMSEA). Como parâmetro os valores de CFI e TLI devem ser acima de 0,90 , os valores de RMSEA devem ser abaixo a 0,08 para indicar ajuste aceitável (Brown, 2015). A consistência interna dos coeficientes da EAP foi testada por meio do alfa de Cronbach com auxílio do IBM-SPSS 22.0. As variáveis com comunalidades $(\leq 0,30)$ e cargas fatoriais $(\leq 0,35)$ abaixo do limite mínimo estabelecido serão excluídas, como também cargas cruzadas com valores símeis em mais de um fator. De modo que, se necessário, outros modelos serão testados.

\section{Resultados}

Inicialmente, buscou-se examinar a estrutura da EAP de acordo com seus pressupostos teóricos e fixou-se seis fatores para análise. A adequabilidade da amostra à AFE foi satisfatória $(\mathrm{KMO}=0,909$; Teste de Esfericidade de Bartlett $\chi^{2}$ [435] 7.469.7; $\left.p<0,001\right)$, com excelentes índices de ajuste $\left[\chi^{2}=27152.374, g l=270 ; \mathrm{CFI}=1,001\right.$; TLI=1,001; RMSEA (IC 90\%)=0,000 (0-0,010)]. Essa solução representa $72,490 \%$ da variância total, com cargas fatoriais que variam em 0,468 e 0,978 e comunalidades entre 0,437 e 0,866 (Tabela 1 ).

Tabela 1

Análise Fatorial Exploratória da Escala de Autonomia Profissional com Seis Fatores

\begin{tabular}{|c|c|c|c|c|c|c|c|}
\hline \multirow{2}{*}{ Itens } & \multicolumn{6}{|c|}{ Fatores } & \multirow{2}{*}{$h^{2}$} \\
\hline & 1 & 2 & 3 & 4 & 5 & 6 & \\
\hline $\begin{array}{l}5 \text { - Tenho liberdade para escolher temáticas } \\
\text { enriquecedoras para os meus alunos, } \\
\text { extraídas de programas oficiais já existentes. }\end{array}$ & 0,978 & $-0,155$ & 0,074 & $-0,049$ & 0,175 & $-0,162$ & 0,866 \\
\hline $\begin{array}{l}22 \text { - Tenho liberdade para escolher temáticas } \\
\text { socioculturais específicas para os meus alunos, } \\
\text { extraídas de programas oficiais já existentes. }\end{array}$ & 0,861 & $-0,110$ & 0,124 & $-0,015$ & 0,144 & $-0,174$ & 0,708 \\
\hline $\begin{array}{l}11 \text { - Tenho liberdade para escolher temáticas } \\
\text { enriquecedoras para os meus alunos, extraídas } \\
\text { de programas elaborados na própria escola. }\end{array}$ & 0,848 & $-0,046$ & $-0,072$ & $-0,051$ & 0,072 & 0,103 & 0,802 \\
\hline $\begin{array}{l}18 \text { - Tenho autonomia para escolher temáticas } \\
\text { enriquecedoras para os meus alunos, } \\
\text { situadas num âmbito extracurricular. }\end{array}$ & 0,720 & 0,098 & 0,079 & $-0,064$ & $-0,038$ & 0.091 & 0,665 \\
\hline $\begin{array}{l}28 \text { - Tenho liberdade para promover intercâmbios } \\
\text { com entidades sociais exteriores à escola. }\end{array}$ & 0,498 & 0,200 & $-0,014$ & 0,118 & $-0,216$ & 0,206 & 0,484 \\
\hline $\begin{array}{l}27 \text { - Tenho autonomia para experimentar } \\
\text { novas formas de apoio educativo. }\end{array}$ & 0,468 & 0,187 & 0,027 & 0,179 & $-0,028$ & 0,112 & 0,578 \\
\hline $\begin{array}{l}6 \text { - Tenho autonomia para acrescentar } \\
\text { ou eliminar temas ao currículo oficial. }\end{array}$ & $-0,055$ & 0,903 & 0,053 & $-0,061$ & 0,039 & $-0,022$ & 0,800 \\
\hline $\begin{array}{l}23 \text { - Tenho liberdade para elaborar um currículo } \\
\text { de acordo com as necessidades dos meus alunos. }\end{array}$ & 0,034 & 0,888 & $-0,018$ & $-0,006$ & $-0,073$ & $-0,055$ & 0,708 \\
\hline $\begin{array}{l}24 \text { - Tenho autonomia para definir os objetivos } \\
\text { curriculares. }\end{array}$ & $-0,038$ & 0,744 & $-0,060$ & 0,032 & 0,154 & 0,039 & 0,672 \\
\hline $\begin{array}{l}30 \text { - Tenho autonomia para experimentar } \\
\text { um novo currículo. }\end{array}$ & $-0,012$ & 0,686 & 0,059 & 0,074 & 0,012 & 0,017 & 0,548 \\
\hline $\begin{array}{l}13 \text { - Tenho autonomia para elaborar } \\
\text { critérios de admissão de alunos. }\end{array}$ & $-0,068$ & 0,047 & 0,893 & $-0,075$ & 0,076 & $-0,119$ & 0,699 \\
\hline $\begin{array}{l}7 \text { - Posso tomar decisões autônomas quanto } \\
\text { à definição de critérios de elaboração de } \\
\text { horário das turmas. }\end{array}$ & 0,142 & $-0,069$ & 0,694 & $-0,159$ & 0,243 & $-0,217$ & 0,437 \\
\hline
\end{tabular}


Tabela 1 (continuação)

Análise Fatorial Exploratória da Escala de Autonomia Profissional com Seis Fatores

\begin{tabular}{|c|c|c|c|c|c|c|c|}
\hline \multirow{2}{*}{ Itens } & \multicolumn{6}{|c|}{ Fatores } & \multirow{2}{*}{$h^{2}$} \\
\hline & 1 & 2 & 3 & 4 & 5 & 6 & \\
\hline $\begin{array}{l}1 \text { - Tenho autonomia para decidir quanto à } \\
\text { definição de critérios de formação de turmas } \\
\text { (turmas heterogêneas ou homogêneas). }\end{array}$ & $-0,045$ & $-0,016$ & 0,680 & 0,099 & $-0,018$ & $-0,007$ & 0,479 \\
\hline $\begin{array}{l}25 \text { - Tenho poder para escolher as condições } \\
\text { do ambiente e espaço físico da sala de aula. }\end{array}$ & 0,088 & $-0,059$ & 0,585 & 0,211 & $-0,020$ & 0,001 & 0,493 \\
\hline $\begin{array}{l}19 \text { - Tenho autonomia para elaborar } \\
\text { normas e regulamentos da escola. }\end{array}$ & 0,214 & 0,058 & 0,529 & $-0,229$ & $-0,082$ & 0,249 & 0,529 \\
\hline $\begin{array}{l}4 \text { - Sinto que posso escolher entre várias } \\
\text { ofertas de formação de âmbito geral, ao } \\
\text { longo do meu desempenho profissional. }\end{array}$ & 0,059 & 0,025 & 0,006 & 0,922 & $-0,078$ & $-0,023$ & 0,837 \\
\hline $\begin{array}{l}26 \text { - Posso escolher o momento em que } \\
\text { pretendo fazer a minha formação profissional. }\end{array}$ & $-0,057$ & 0,037 & 0,041 & 0,903 & 0,000 & $-0,103$ & 0,744 \\
\hline $\begin{array}{l}17 \text { - Sinto que posso escolher entre várias } \\
\text { ofertas de formação, que respondam às minhas } \\
\text { necessidades sentidas na série que atuo. }\end{array}$ & $-0,008$ & $-0,061$ & $-0,085$ & 0,880 & 0,066 & 0,055 & 0,797 \\
\hline $\begin{array}{l}10 \text { - Posso escolher entre várias ofertas de } \\
\text { formação de âmbito específica do meu curso, } \\
\text { ao longo do meu desempenho profissional. }\end{array}$ & 0,089 & 0,037 & $-0,007$ & 0,776 & $-0,016$ & 0,009 & 0,687 \\
\hline $\begin{array}{l}\text { 21- Posso escolher o local da } \\
\text { minha formação profissional. }\end{array}$ & 0,027 & $-0,062$ & 0,009 & 0,690 & 0,053 & $-0,053$ & 0,461 \\
\hline $\begin{array}{l}29 \text { - Tenho poder para recorrer a diferentes } \\
\text { formas de avaliação dos alunos. }\end{array}$ & 0,029 & 0,051 & $-0,012$ & $-0,052$ & 0,841 & $-0,053$ & 0,706 \\
\hline $\begin{array}{l}2 \text { - Tenho autonomia quanto ao trabalho } \\
\text { a realizar dentro da sala de aula. }\end{array}$ & 0,159 & 0,011 & $-0,081$ & $-0,046$ & 0,801 & $-0,041$ & 0,752 \\
\hline $\begin{array}{l}14 \text { - Tenho autonomia para decidir quanto } \\
\text { aos meios e aos procedimentos para } \\
\text { avaliar o rendimento escolar dos alunos. }\end{array}$ & 0,097 & 0,128 & $-0,072$ & $-0,036$ & 0,785 & $-0,185$ & 0,683 \\
\hline $\begin{array}{l}8 \text { - Tenho poder para determinar as normas } \\
\text { e as regras de comportamento dos alunos. }\end{array}$ & $-0,181$ & 0,046 & 0,075 & 0,114 & 0,717 & 0,088 & 0,561 \\
\hline $\begin{array}{l}31 \text { - Tenho poder para estabelecer } \\
\text { limites ao comportamento dos alunos. }\end{array}$ & $-0,191$ & $-0,094$ & 0,168 & 0,105 & 0,682 & 0,186 & 0,527 \\
\hline $\begin{array}{l}12 \text { - Tenho autonomia para definir a ordem de } \\
\text { apresentação dos temas/conteúdos curriculares. }\end{array}$ & 0,039 & 0,222 & $-0,018$ & $-0,031$ & 0,628 & 0,048 & 0,646 \\
\hline $\begin{array}{l}15 \text { - Tenho autonomia para experimentar } \\
\text { novos métodos de ensino. }\end{array}$ & 0,082 & 0,253 & $-0,036$ & $-0,031$ & 0,555 & 0,058 & 0,623 \\
\hline $\begin{array}{l}16 \text { Tenho poder para promover atividades culturais } \\
\text { com os pais ou encarregados de educação. }\end{array}$ & $-0,007$ & 0,022 & $-0,007$ & $-0,005$ & $-0,073$ & 0,907 & 0,764 \\
\hline $\begin{array}{l}9 \text { - Tenho poder para promover encontros } \\
\text { com os pais ou encarregados de educação, } \\
\text { para tratar assuntos escolares. }\end{array}$ & $-0,036$ & $-0,032$ & $-0,015$ & 0,059 & 0,099 & 0,836 & 0,757 \\
\hline $\begin{array}{l}3 \text { - Tenho autonomia para desencadear } \\
\text { formas de colaboração com os pais } \\
\text { ou encarregados de educação. }\end{array}$ & 0,057 & $-0,088$ & $-0,012$ & 0,066 & 0,088 & 0,813 & 0,786 \\
\hline Eigenvalues & 11,716 & 2,984 & 2,595 & 1,863 & 1,385 & 1,201 & \\
\hline \% var, explicada & 39,054 & 9,947 & 8,652 & 6,212 & 4,619 & 4,005 & \\
\hline Análise Paralela - \% var. explicada & $43,094^{*}$ & $10,704^{*}$ & $9,118^{*}$ & 6,496 & 4,811 & 4,251 & \\
\hline$\alpha$ & 0,89 & 0,86 & 0,73 & 0,89 & 0,89 & 0,89 & \\
\hline$\chi^{2}$ & & & & 7152.374 & & & \\
\hline $\mathrm{Gl}$ & & & & 270 & & & \\
\hline CFI & & & & 1.001 & & & \\
\hline TLI & & & & 1.001 & & & \\
\hline RMSEA (IC 90\%) & & & 0,00 & $0(0-0,0$ & & & \\
\hline
\end{tabular}

Nota. ${ }^{*}$ Número recomendado de dimensões: $3 ; \chi^{2}=$ qui-quadrado; $g l=$ graus de liberdade 
A estrutura de seis fatores (F1. Autonomia na escolha de temáticas, $\alpha=0,89 ; \mathrm{F} 2$. Autonomia sobre o currículo, $\alpha=0,86 ;$ F3. Autonomia na formação de turmas, $\alpha=0,73$; F4. Autonomia na formação profissional, $\alpha=0,89$; F5. Autonomia na avaliação do rendimento escolar, $\alpha=0,89$; e F6. Autonomia no relacionamento com os responsáveis pela educação, $\alpha=0,89$ ) apresentou consistência interna adequada. Entretanto, a AP sugeriu uma estrutura tridimensional e seguiu-se à verificação (Tabela 1).

A amostra foi submetida a mais uma AFE na qual fixou-se o número de fatores em três. $\mathrm{O}$ item $\mathrm{n}^{\mathrm{o}}$
7 apresentou comunalidade $\left(h^{2}=0,265\right)$ abaixo do limite mínimo estabelecido. O item $\mathrm{n}^{\mathrm{o}} 28$ manteve carga cruzada símil e insignificativa em dois fatores $(\lambda=0.309 ; \lambda=0.336)$. Assim, essas duas variáveis foram excluídas e uma estrutura fatorial composta por 28 itens distribuídos em três fatores se mostrou adequada $\left(\mathrm{KMO}=0,908 ;\right.$ Teste de Esfericidade de Bartlett $\chi^{2}[378]$ 7.077.0; $p<0,001)$, com índices de ajustes satisfatórios $\left[\chi^{2}=24666.361, g l=297 ; \mathrm{CFI}=0.967 ; \mathrm{TLI}=0.974\right.$; RMSEA $(\mathrm{IC} 90 \%)=0.073(0.0629-0.0797)]$. Essa solução fatorial explica $59,306 \%$ da variância total e a AP representa $65,689 \%$ (Tabela 2 ).

Tabela 2

Análise Fatorial Exploratória da Escala de Autonomia Profissional com Três Fatores

\begin{tabular}{|c|c|c|c|c|}
\hline \multirow{2}{*}{ Itens } & \multicolumn{3}{|c|}{ Fatores } & \multirow{2}{*}{$h^{2}$} \\
\hline & 1 & 2 & 3 & \\
\hline $\begin{array}{l}17 \text { - Sinto que posso escolher entre várias ofertas de formação, } \\
\text { que respondam às minhas necessidades sentidas na série que atuo. }\end{array}$ & 0,890 & 0,009 & $-0,023$ & 0,779 \\
\hline $\begin{array}{l}4 \text { - Sinto que posso escolher entre várias ofertas de formação } \\
\text { de âmbito geral, ao longo do meu desempenho profissional. }\end{array}$ & 0,872 & 0,135 & $-0,100$ & 0,780 \\
\hline $\begin{array}{l}26 \text { - Posso escolher o momento em que pretendo } \\
\text { fazer a minha formação profissional. }\end{array}$ & 0,829 & 0,069 & $-0,102$ & 0,658 \\
\hline $\begin{array}{l}10 \text { - Posso escolher entre várias ofertas de formação de âmbito } \\
\text { específica do meu curso, ao longo do meu desempenho profissional. }\end{array}$ & 0,752 & 0,122 & 0,006 & 0,659 \\
\hline 21 - Posso escolher o local da minha formação profissional. & 0,696 & $-0,022$ & $-0,051$ & 0,442 \\
\hline 19 - Tenho autonomia para elaborar normas e regulamentos da escola. & $-0,172$ & 0,778 & $-0,037$ & 0,501 \\
\hline 13 - Tenho autonomia para elaborar critérios de admissão de alunos. & $-0,053$ & 0,757 & $-0,219$ & 0,406 \\
\hline $\begin{array}{l}1 \text { - Tenho autonomia para decidir quanto à definição de critérios } \\
\text { de formação de turmas (turmas heterogêneas ou homogêneas). }\end{array}$ & 0,120 & 0,683 & $-0,292$ & 0,359 \\
\hline $\begin{array}{l}25 \text { - Tenho poder para escolher as condições } \\
\text { do ambiente e espaço físico da sala de aula. }\end{array}$ & 0,257 & 0,657 & $-0,239$ & 0,443 \\
\hline $\begin{array}{l}16 \text { - Tenho poder para promover atividades } \\
\text { culturais com os pais ou encarregados de educação. }\end{array}$ & 0,078 & 0,572 & 0,096 & 0,451 \\
\hline $\begin{array}{l}3 \text { - Tenho autonomia para desencadear formas de } \\
\text { colaboração com os pais ou encarregados de educação. }\end{array}$ & 0,201 & 0,475 & 0,187 & 0,520 \\
\hline $\begin{array}{l}9 \text { - Tenho poder para promover encontros com os pais } \\
\text { ou encarregados de educação, para tratar assuntos escolares. }\end{array}$ & 0,166 & 0,474 & 0,190 & 0,489 \\
\hline $\begin{array}{l}14 \text { - Tenho autonomia para decidir quanto aos meios e aos } \\
\text { procedimentos para avaliar o rendimento escolar dos alunos. }\end{array}$ & 0,031 & $-0,361$ & 0,957 & 0,656 \\
\hline 2 - Tenho autonomia quanto ao trabalho a realizar dentro da sala de aula. & 0,090 & $-0,293$ & 0,942 & 0,716 \\
\hline 29 - Tenho poder para recorrer a diferentes formas de avaliação dos alunos. & 0,059 & $-0,256$ & 0,902 & 0,648 \\
\hline $\begin{array}{l}12 \text { - Tenho autonomia para definir a ordem de } \\
\text { apresentação dos temas/conteúdos curriculares. }\end{array}$ & $-0,005$ & $-0,091$ & 0,849 & 0,634 \\
\hline 15 - Tenho autonomia para experimentar novos métodos de ensino. & $-0,024$ & $-0,059$ & 0,834 & 0,622 \\
\hline 24 - Tenho autonomia para definir os objetivos curriculares. & $-0,210$ & 0,152 & 0,699 & 0,512 \\
\hline 6 - Tenho autonomia para acrescentar ou eliminar temas ao currículo oficial. & $-0,361$ & 0,294 & 0,636 & 0,532 \\
\hline 8 - Tenho poder para determinar as normas e as regras de comportamento dos alunos. & 0,186 & $-0,079$ & 0,621 & 0,470 \\
\hline $\begin{array}{l}11 \text { - Tenho liberdade para escolher temáticas enriquecedoras para } \\
\text { os meus alunos, extraídas de programas elaborados na própria escola. }\end{array}$ & 0,128 & 0,143 & 0,597 & 0,585 \\
\hline $\begin{array}{l}5 \text { - Tenho liberdade para escolher temáticas enriquecedoras } \\
\text { para os meus alunos, extraídas de programas oficiais já existentes. }\end{array}$ & 0,174 & 0,117 & 0,593 & 0,597 \\
\hline $\begin{array}{l}23 \text { - Tenho liberdade para elaborar um currículo } \\
\text { de acordo com as necessidades dos meus alunos. }\end{array}$ & $-0,320$ & 0,272 & 0,580 & 0,448 \\
\hline $\begin{array}{l}22 \text { - Tenho liberdade para escolher temáticas socioculturais específicas } \\
\text { para os meus alunos, extraídas de programas oficiais já existentes }\end{array}$ & 0,168 & 0,154 & 0,511 & 0,513 \\
\hline
\end{tabular}


Tabela 2 (continuação)

Análise Fatorial Exploratória da Escala de Autonomia Profissional com Três Fatores

\begin{tabular}{|c|c|c|c|c|}
\hline \multirow{2}{*}{ Itens } & \multicolumn{3}{|c|}{ Fatores } & \multirow{2}{*}{$h^{2}$} \\
\hline & 1 & 2 & 3 & \\
\hline 30 - Tenho autonomia para experimentar um novo currículo. & $-0,173$ & 0,298 & 0,486 & 0,402 \\
\hline $\begin{array}{l}18 \text { - Tenho autonomia para escolher temáticas enriquecedoras } \\
\text { para os meus alunos, situadas num âmbito extracurricular. }\end{array}$ & 0,030 & 0,333 & 0,471 & 0,542 \\
\hline 31 - Tenho poder para estabelecer limites ao comportamento dos alunos. & 0,231 & 0,060 & 0,450 & 0,406 \\
\hline 27 - Tenho autonomia para experimentar novas formas de apoio educativo. & 0,189 & 0,280 & 0,400 & 0,525 \\
\hline Eigenvalues & 2,425 & 2,983 & 11,196 & \\
\hline$\%$ var. explicada & 8,663 & 10,655 & 39,988 & \\
\hline Análise Paralela - \% var. explicada & 9,250 & 11,631 & 44,806 & \\
\hline$\alpha$ & 0,89 & 0,80 & 0,92 & \\
\hline$\chi^{2}$ & \multicolumn{4}{|c|}{24666.361} \\
\hline $\mathrm{Gl}$ & \multicolumn{4}{|c|}{297} \\
\hline CFI & \multicolumn{4}{|c|}{0,967} \\
\hline TLI & \multicolumn{4}{|c|}{0,974} \\
\hline RMSEA (IC 90\%) & \multicolumn{4}{|c|}{$0,073(0,0629-0,0797)$} \\
\hline
\end{tabular}

O modelo tridimensional apresentou comunalidades que oscilam em 0,359 e 0,780 e dividiu-se em: F1. Autonomia na formação profissional (5 itens, $\alpha=0,89$ ); F2. Autonomia sobre o currículo e relacionamento com os responsáveis da educação (7 itens, $\alpha=0,80$ ); e F3. Autonomia sobre o ensino (16 itens, $\alpha=0,92)$, conforme pode ser observado na Tabela 2 .

\section{Discussão}

A AEP mostrou-se adequada para averiguação da intensidade da autonomia do professorado, mas dois modelos foram apresentados. A solução com seis fatores mostrou evidências de validade e consistência interna semelhante a versão anterior (Veiga et al., 2003). O modelo tridimensional sugerido pela AP manteve índices de ajustamento satisfatórios, porém o fator Autonomia sobre o ensino aglutinou 16 itens, tornando sua interpretação abstrusa.

A variância total foi mais bem explicada pela solução de seis fatores, já que 72,490\% é representada pelos dados trazidos na matriz fatorial. E o índice para esse modelo é alto, mostrando a estreita relação entre os itens, em comparação à solução tridimensional, com 59,306\% (Hair Jr. et al., 2009).

A consistência interna testada pelo alfa de Cronbach mostrou-se satisfatória nos dois modelos propostos. Porém, os fatores Autonomia na escolha de temáticas, Autonomia na formação profissional, Autonomia na avaliação do rendimento escolar e Autonomia no relacionamento com os responsáveis pela educação, encontradas na estrutura de seis fatores apresentaram ótima consistência, com alfa de 0,89 . O fator Autonomia sobre o ensino $(\alpha=0,92)$ do modelo tridimensional revelou redundância ou duplicação, já que valores acima de 0,90 mostram que itens do mesmo fator estão mensurando exatamente o mesmo objeto (Oviedo \& Campo-Arias, 2005).

O modelo composto por seis dimensões se mostrou bastante ajustado e isso ocorre porque quanto maior o número de fatores explicando a variância dos dados, menos resíduos são gerados. Para tanto, índices de ajuste não podem ser considerados um critério para retenção fatorial (Brown, 2015). Por sua vez, a AP é empregada para verificação de demissões, pois reduz a probabilidade da retenção, muitas vezes, superestimada de itens (Damásio, 2012).

No nosso caso, a solução tridimensional ficou inadequada, enquanto a de seis fatores apresentou superajustada, mostrando como o modelo que favorecerá uma melhor explicação para a percepção da autonomia dos professores no contexto brasileiro. Mesmo assim, fazem-se necessários novos estudos para averiguar a melhor solução fatorial. A utilização da análise fatorial confirmatória multigrupos com a participação de professores das escolas privadas e de outras esferas é imprescindível. Uma vez que tal técnica possibilita a avaliação da configuração e das medidas de determinado instrumento em grupos heterogêneos simultaneamente (Damásio, 2013). Também é propício correlacionar a EAP com outros instrumentos, com a finalidade de realizar a validação convergente (Freitas \& Damásio, 2017; Hair et al., 2009), tendo em vista a complexidade de tal fenômeno, a qual desempenha um papel decisivo na legitimação do status docente, na aprendizagem online, nas tomadas de decisões na escola, como também contribui com uma atmosfera cooperativa, o desenvolvimento profissional do professor, entre outras (Derakhshan et al., 2020; Evers et al., 2017; Vangrieken et al., 2017; Worth \& Brande, 2020). 
Apesar de envolver diferentes níveis de ensino, a principal limitação do presente estudo é não se basear em uma amostra brasileira representativa, tendo em vista que foi aplicada em professores de uma única cidade, sendo de extrema importância uma população maior para fins de replicação. No entanto, este estudo permitiu explorar a autonomia profissional dos professores da cidade de Mossoró, dado a complexidade de tal fenômeno. A EAP ganha proeminência ao corroborar a autonomia destacando os impasses, situações e condições vivenciadas no contexto de trabalho do professor, fornecendo aos formuladores de políticas públicas educacionais e gestores escolares informações para melhorar o contexto de trabalho docente e, consequentemente, a qualidade do ensino.

\section{Agradecimentos}

Não há menções.

\section{Financiamento}

A presente pesquisa não recebeu nenhuma fonte de financiamento sendo custeada com recursos dos próprios autores.

\section{Contribuições dos autores}

Declaramos que todos os autores participaram da elaboração do manuscrito. Especificamente, o(s) autor(es) Silvia Fernandes do Vale e Regina Heloisa Mattei de Oliveira Maciel participou(ram) da redação inicial do estudo - conceitualização, investigação, visualização, o(s) autor(es) Silvia Fernandes do Vale, Regina Heloisa Mattei de Oliveira Maciel e Mateus Estevam Medeiros Costa participou(ram) da análise dos dados, e o(s) autor(es) Silvia Fernandes do Vale, Regina Heloisa Mattei de Oliveira Maciel e Mateus Estevam Medeiros Costa participou(ram) da redação final do trabalho - revisão e edição.

\section{Disponibilidade dos dados e materiais}

Todos os dados e sintaxes gerados e analisados durante esta pesquisa serão tratados com total sigilo devido às exigências do Comitê de Ética em Pesquisa em Seres Humanos. Porém, o conjunto de dados e sintaxes que apoiam as conclusões deste artigo estão disponíveis mediante razoável solicitação ao autor principal do estudo.

\section{Conflito de interesses}

Os autores declaram que não há conflitos de interesses.

\section{Referências}

Brown, T. A. (2015). Confirmatory factor analysis for applied research. The Guilford Press.

Borsa, J. C., Damásio, B. F., \& Bandeira, D. R. (2012). Adaptação e validação de instrumentos psicológicos entre culturas: Algumas considerações. Paidéia (Ribeirão Preto), 22(53), 423-432. https://doi.org/10.1590/S0103-863X2012000300014.

Borsa, J. C., \& Seize, M. M. (2017). Construção e adaptação de instrumentos psicológicos: dos caminhos possíveis. Em B. F. Damásio \& J. C. Borsa. (Orgs.). Manual de desenvolvimento de instrumentos psicológicos. (1ed., pp. 15-37). Vetor.

Charters Jr., W. W. (1976). Sense of teacher work autonomy: Measurement and findings. Eugene. Center for Educational Policy and Management, University of Oregon.

Chauvin. S. W., \& Ellett. C. D. (1993). Teachers' professional orientation: An empirical examination of the construct using the results of large-scale factor analysis. Paper presented at the annual meeting of the Mid-South Educational Research Association. New Orleans. Louisiana.

Contreras, J. (2002). Autonomia de professores. Cortez.

Damásio. B. F. (2012). Uso da análise fatorial exploratória em psicologia. Avaliação Psicológica. 11(2), 213-228. http://pepsic.bvsalud.org/ scielo.php?script $=$ sci arttext\&pid $=$ S1677-04712012000200007\&lng $=$ pt\&tlng $=p t$

Damásio, B. F. (2013). Contribuições da Análise Fatorial Confirmatória Multigrupo (AFCMG) na avaliação de invariância de instrumentos psicométricos. Psico-USF, 18(2), 211-220. https://www.redalyc.org/pdf/4010/401036095005.pdf

Damásio. B. F., \& Dutra. D. F. (2017). Análise fatorial exploratória: Um tutorial com o software fator. Em B. F. Damásio \& J. C. Borsa. (Orgs.), Manual de desenvolvimento de instrumentos psicológicos. (1a ed., pp. 241-265).Vetor.

Derakhshan, A, Coombe, C., Arabmofrad, A., \& Taghizadeh, M. (2020) Investigating the effects of english language teachers' professional identity and autonomy in their success. Issues in Language Teaching, 9(1), 1-28. https://doi.org/0.22054/ilt.2020.52263.496.

Erss, M., Kalmus, V., \& Autio, T. H. (2016). Walking a fine line: Teachers perception of curricular autonomy in Estonia, Finland and Germany. Journal of Curriculum Studies, 48(5), 589-609. https://doi.org/10.1080/00220272.2016.1167960

Evers, A. T., Verboon, P., \& Klaeijsen, A. (2017). The development and validation of a scale measuring teacher autonomous behaviour. British Educational Research Journal, 43(4), 805-821. https://doi.org/10.1002/berj.3291

Fernandes, M. J. D. S., \& Barbosa, A. (2014). O trabalho docente na rede pública do estado de São Paulo: Apontamentos iniciais para a discussão da jornada de trabalho. Práxis Educacional, 10(17), 117-142. https://repositorio.unesp.br/bitstream/handle/11449/125053/ ISSN2178-2679-2014-10-17-117-142.pdf?sequence =1\&isAllowed =y

Ferreira. M. C. (2008). Chegar feliz e sair feliz do trabalho: aportes do reconhecimento no trabalho para uma ergonomia aplicada à qualidade de vida no trabalho. Em A. M. Mendes. (Org.). Trabalho e Saúde: O Sujeito entre Emancipação e Servidão (pp. 40-53). Juruá.

Filho, A. A. (2020). Atividade, significação e sentido: Bases do sofrimento psicológico e a especificidade do adoecimento do professor. Em M. G. D. Facci \& S. C. Urt (Orgs.). Quando os professores adoecem. (pp. 73-104). Ed. UFMS.

Freire, P. (1996). Pedagogia da autonomia: Saberes necessários à prática educativa. Paz e Terra.

Freitas, C. P. P., \& Damásio, B. F. (2017). Evidências de validade com base nas relações com medidas externas: Conceituação e problematização, Em B. F. Damásio \& J. C. Borsa. (Orgs.). Manual de desenvolvimento de instrumentos psicológicos. (1a ed., pp. 101-117). Vetor. 
Forsyth. P. B., \& Danisiewicz. T. J. (1985). Toward a theory of professionalization. Work and occupations. 12(1), 59-76. https://doi.org/10.117 7\%2F0730888485012001004

Friedman. I. A. (1999). Teacher-perceived work autonomy: The concept and its measurement. Educational and psychological Measurement. 59(1). 58-76. https://doi.org/10.1177\%2F0013164499591005

Hair, J. F., Black, W. C., Babin, B. J., \& Anderson, R. E. (2009). Multivariate Data Analysis. (7th Eds.) Pearson.

Khezerlou. E. (2013). Teacher Autonomy Perceptions of Iranian and Turkish EFL Teachers. Journal of History Culture and Art Research, 2(2), 199-211. https://doi.org/10.7596/taksad.v2i2.224

Lessard. C. (2006). Multiple regulation and teacher's professional autonomy: Comparisons between Quebec and Canada. Educação em Revista,44, 143-163. https://doi.org/10.1590/S0102-46982006000200008

Lorenzo-Seva, U. (2013). Why rotate my data using Promin? Technical Report. Department of Psychology, Universitat Rovira i Virgili, Tarragona.

Martins. M. F. D., Vieira. J. S., Feijó. J. R., \& Bugs. V. (2014). O trabalho das docentes da Educação Infantil e o mal-estar docente: O impacto dos aspectos psicossociais no adoecimento. Cadernos de Psicologia Social do Trabalho, 17(2), 281-289. http://www.periodicos.usp.br/cpst/ article/view/112348/110312

Moraes. R. D., Vasconcelos. A. C. L., \& Cunha. S. C. P. (2012). Prazer no trabalho: O lugar da autonomia. Revista Psicologia Organizações e Trabalho. 12(2), 217-227. http://pepsic.bvsalud.org/scielo.php?script=sci_arttext\&pid =S1984-66572012000200007\&lng=pt\&tlng=pt

Nayernia, A. (2020). Development and validation of an e-teachers' autonomy-support scale: A SEM approach. International Jounal of Language Studies, 14(2), 117-134. https://www.researchgate.net/profile/Akram-Nayernia/publication/339933993 Development and validation of_an_e-teachers'_autonomy-support_scale_A_SEM_approach/links/5e6cfeb992851c6ba703ddd3/Development-and-validation-ofan-e-teachers-autonomy-support-scale-A-SEM-approach.pdf

Oviedo, H. C., \& Campo-Arias, A. (2005). Aproximación al uso del coeficiente alfa de Cronbach. Revista Colombiana de Psiquiatría, 34(4), 527-580. https://www.redalyc.org/pdf/806/80634409.pdf

Pearson. L. C., \& Moomaw. W. (2005). The relationship between teacher autonomy and stress. work satisfaction. empowerment. and professionalism. Educational research quarterly. 29(1), 38-54. https://files.eric.ed.gov/fulltext/EJ718115.pdf

Przenyczka. R. A., Lenardt. M. H., Mazza. V. A., \& Lacerda. M. R. (2012). The paradox of freedom and autonomy in nurses' actions. Texto E Contexto - Enfermagem. 21(2), 427-431. https://doi.org/10.1590/S0104-07072012000200022

Santos. F. D. O. F. D. Montezeli. J. H., \& Peres. A. M. (2012). Autonomia profissional e sistematização da assistência de enfermagem: Percepção de enfermeiros. Revista Mineira de Enfermagem, 16(2), 251-257. http://reme.org.br/artigo/detalhes/526

Skaalvik, E. M., \& Skaalvik, S. (2014). Teacher Self-Efficacy and Perceived Autonomy: Relations with Teacher Engagement, Job Satisfaction, and Emotional Exhaustion. Psychological Reports, 114(1), 68-77. https://doi.org/10.2466\%2F14.02.PR0.114k14w0

Souza. D. C. C., Fernandes. B. P., \& Filgueira. V. (2015). Racionalização, intensidade e controle do trabalho docente na rede básica de ensino. Trabalho E Educação, 24(3), 123-141. https://periodicos.ufmg.br/index.php/trabedu/article/view/9456/6749

Timmerman. M. E., \& Lorenzo-Seva. U. (2011). Dimensionality assessment of ordered polytomous items with parallel analysis. Psychological Methods.16(2), 209. https://doi.org/10.1037/a0023353

Ulas. J., \& Aksu. M. (2015). Development of teacher autonomy scale for turkish teachers. Procedia-Social and Behavioral Sciences, 186, $344-349$. https://doi.org/10.1016/j.sbspro.2015.04.023

Vangrieken, K., Grosemans, I., Dochy, F., \& Kyndt, E. (2017). Teacher autonomy and collaboration: A paradox? Conceptualising and measuring teachers' autonomy and collaborative attitude. Teaching and Teacher Education, 67, 302-315. http://www.evakyndt.com/ uploads/1/2/5/2/125271798/vangrieken_grosemans_dochy_kyndt_2017.pdf

Veiga. F., Guerra. T. M., Fernandes. L., Roque. P., \& Antunes. J. (2003). Escala de avaliação da autonomia profissional dos professores: Elaboração e validação. Revista Galego-Portuguesa de Psicoloxía e Educación, 8(10), 1009-1017. https://repositorio.ul.pt/ bitstream/10451/4676/1/Escala\%20de\%20avalia\%c3\%a7\%c3\%a3o\%20da\%20autonomia\%20profissional\%20dos\%20professoresElabora\%c3\%a7\%c3\%a3o\%20e\%20valida\%c3\%a7\%c3\%a3o.pdf

Yu-hong. J. I. A. N. G., \& Ting. M. (2012). a comparative study of teacher autonomy between novice teachers and proficient teachers in the context of university english teaching reform in China. Sino-US English Teaching, 9(3), 963-974. http://www.airitilibrary.com/ Publication/alDetailedMesh?docid=15398072-201203-201207200008-201207200008-963-974

Wilches. J. U. (2009). Teacher autonomy: A critical review of the research and concept beyond applied linguistics. Íkala, 12(1), 245-275. http://www.redalyc.org/pdf/2550/255020488010.pdf

Worth, J., \& Brand, V. (2020). Teacher autonomy: How does it related to job satisfaction and retention? National Foudation for Educational Research. Slough: NFER. https://files.eric.ed.gov/fulltext/ED604418.pdf

\section{Sobre os autores}

Silvia Fernandes do Vale é doutora em Psicologia pela Universidade de Fortaleza (UNIFOR). Atualmente, é Professora da Rede Municipal de Ensino de Mossoró (RN), pesquisadora do Laboratório de Estudos sobre o Trabalho (LET/UNIFOR).

Regina Heloisa Mattei de Oliveira Maciel é doutora em Psicologia pela Universidade de São Paulo. Atualmente é professora no Programa de Pós-Graduação Stricto Sensu em Psicologia da Universidade de Fortaleza (UNIFOR). Coordenadora do Laboratório de Estudos sobre o Trabalho (LET/UNIFOR).

Mateus Estevam Medeiros Costa é doutorando em Psicologia pela Universidade Federal do Rio Grande do Norte (UFRN). Pesquisador do Grupo de Estudos e Pesquisas sobre o Trabalho (GEPET-UFRN).

\section{Como citar este artigo}

Vale, S. F., Maciel, R. H., Medeiros-Costa, M. E. (2021) Escala de Avaliação da Autonomia Profissional dos Professores: adaptação e propriedades psicométricas. Avaliação Psicológica, 20(3), 352-360. http://dx.doi.org/10.15689/ap.2021.2003.18741.09 